\title{
Origin of ventricular reflexes caused by coronary arteriography
}

\author{
F. PEREZ-GOMEZ AND A. GARCIA-AGUADO \\ From the Departamento Cardiopulmonar, Hospital Clinico de la Facultad de Medicina, Madrid, Spain
}

Left ventricular reflexes have in the past been investigated in anaesthetised animals, generally using an open chest technique. We have studied the degree of bradycardia occurring during coronary arteriography in 200 patients with a view to localising the origin of the ventricular reflexes. We have correlated the decrease of sinus rate with the anatomical distribution and integrity of the coronary tree.

The degree of bradycardia was not influenced by the origin of the sinus node or the AV node arteries, while there was a good correlation with the injection of contrast medium into the artery which supplied the inferior wall of the left ventricle. The occurrence of transient sinus arrest was also correlated with the injection into the same artery.

The results suggest that the parasympathetic receptors are located mainly in the inferior wall of the left ventricle. This may well be the explanation for the clinical picture of bradycardia, hypotension, and peripheral vasodilatation often seen in acute inferior myocardial infarction.

Several experimental studies have been devoted to ischaemic myocardial reflexes. Some were performed by ligature of the coronary arteries (Hanley et al., 1971, 1972), others by injection of microspheres of mercury into the aortic root (Agress et al., 1957). In others the mercury was selectively injected into the circumflex artery. Increase of intraventricular pressure, resulting from an increase of afterload, causes reflex responses similar to those resulting from ischaemia (Sleight and Widdicombe, 1965; Öberg and Thorén, 1972). These experiments were performed in anaesthetised animals, in most cases with an open chest, and are therefore not necessarily applicable to man. The conclusions drawn were concerned with the reflex response, not the origin of the reflexes, as pointed out by Linden (1975) after a thorough review of the published reports.

Coronary arteriography has been found to cause reflex responses similar to those occurring in ischaemia. Among others, Carson and Lazzara (1970) reported bradycardia, hypotension, and peripheral vasodilatation during coronary arteriography, but no attempt was made to localise the area from which these reflexes originated.

In this study we have evaluated the degree of bradycardia during coronary arteriography in an attempt to localise the origin of the ventricular reflexes in man.

Received for publication 25 February 1977

\section{Material and methods}

We have reviewed the electrocardiographic and angiographic data of 200 unselected patients in whom coronary arteriography was performed. One hundred and seventy of the patients were men and the remaining 30 were women. The age range was from 22 to 67 years for the women and from 17 to 74 for the men. The final diagnosis was ischaemic heart disease in $119(59.5 \%)$, rheumatic valvular disease in $35(17.5 \%)$, congestive cardiomyopathy in $21(10.5 \%)$, and hypertrophic cardiomyopathy in 7. Twenty-three were normal and other diagnoses were made in the 5 remaining cases.

The patients were premedicated half an hour before the investigation with an intramuscular injection of $5 \mathrm{mg}$ diazepam, $0.5 \mathrm{mg}$ atropine, and $25 \mathrm{mg}$ promethazine. Left ventriculography was performed in a 45 degree right anterior projection and left ventricular pressures were recorded before and after angiocardiography. Coronary arteriography was performed using multiple projections with $35 \mathrm{~mm}$ cine film at 60 frames per second. Urografin $76(5.9 \mathrm{ml})$ was injected selectively into the main trunk of the coronary artery. The technique described by Sones and Shirey (1962) was used in 55 cases and that of Judkins (1968) in the others. One or two electrocardiographic leads were recorded during angiography.

The coronary arteriograms were assessed in 

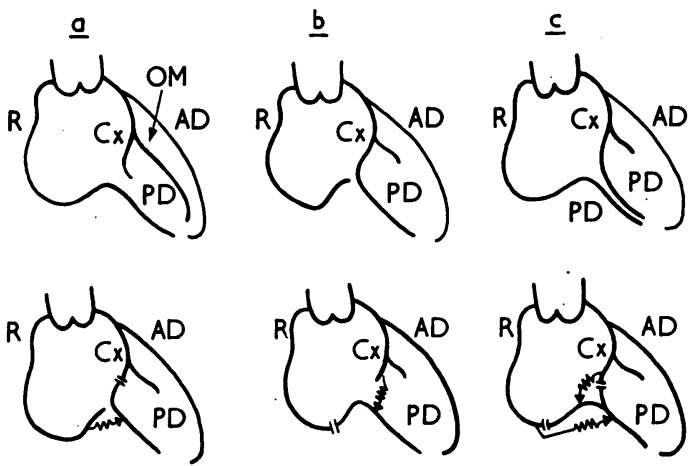

$\rightarrow-$ Obstruction

- Collateral circulation
Fig. 1 Perfusion of the inferior wall of the left ventricle: (a) from the right coronary, (b) from the left and (c) balanced circulation. $R$, right coronary artery. Cx, circumflex; $A D$, anterior descending; $P D$, posterior descending; OM, obtuse marginal

respect of the following features: (1) origin of the sinus node artery; (2) origin of the AV node artery; (3) any obstruction greater than 50 per cent in the lumen of the vessels; and (4) which artery played the predominant role in the perfusion of the inferior wall of the left ventricle. The dominant artery was considered to be that which gave origin to the posterior descending artery and to the atrioventricular node artery (Fig. 1a, b), but the contralateral vessel was considered dominant (Fig. la and b, lower side) when it supplied the posterior descending artery because of obstruction of the dominant artery. We considered that the circulation was balanced when each artery gave rise to a posterior descending artery or when there was severe obstruction of the right coronary artery and of the circumflex artery (Fig. 1, right).

We measured the slowing of the sinus rate during injection of each coronary artery and we used the greatest decrease achieved for each coronary arterial injection (longest PP interval) in our statistical studies. We also noted the incidence of sinus arrest for more than $2 \mathrm{~s}$. The analysis of the coronary arteriograms and of the left ventriculograms and pressures was done independently of the electrocardiograms and the results were given separately for statistical analysis. We compared the decrease of sinus rate during right coronary arteriography with that produced during left coronary arteriography, taking into consideration their angiographic distribution.

In order to clarify some of the findings of the above-mentioned study we surveyed our files of normal coronary angiograms in which the right coronary artery gave origin to one or two posterior descending arteries and we separated the cases into two groups: one with balanced circulation to the inferior wall of the left ventricle (26 cases) in which the circumflex artery and the obtuse marginal artery supplied a large area of that wall and the other with obvious right dominance ( 25 cases) in which the circumflex artery was poorly developed and the obtuse marginal artery followed a lateral pathway.

\section{Results}

The right coronary artery gave origin to the sinus node artery in 119 cases $(65.4 \%)$ and the left coronary artery did so in $63(34.6 \%)$. In the remaining cases the origin of this artery could not be ascertained. The atrioventricular node artery originated from the right coronary artery in 167 cases $(83.5 \%)$ and from the left coronary artery in $23(11.5 \%)$. In 10 cases the origin of this artery could not be identified.

The right coronary artery was dominant, according to the classic definition, in 139 cases $(69.5 \%)$ and the left coronary artery was dominant in 37 cases. The circulation was balanced in 21 cases

Table 1 Comparison of decrease in sinus rate during left and right coronary arteriography in various groups of patients

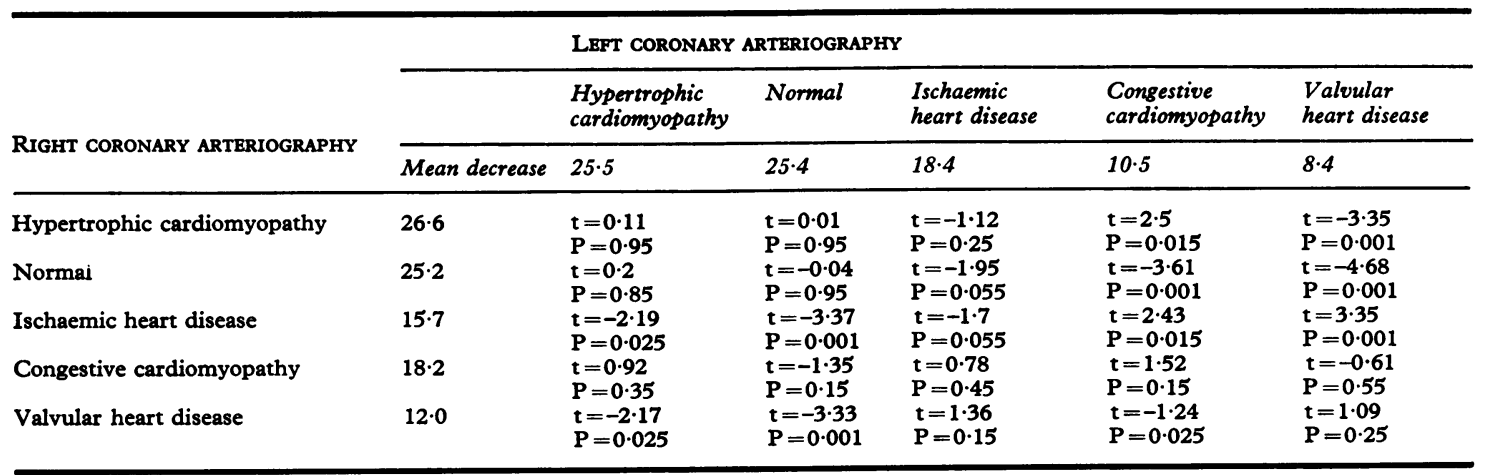




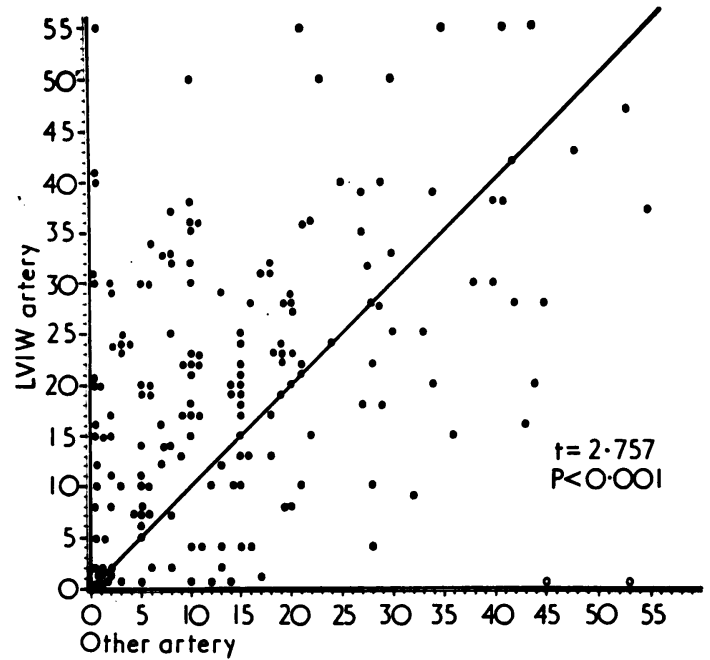

Fig. 2 Comparative results of the decrease of sinus rate in beats per min during injection of the artery which provides the major part of the perfusion of the inferior wall of the left ventricle (LVIW artery) and during injection of the other artery. The line of identity is shown.

$(10.5 \%)$ and we could not determine the dominance in the remaining 3 cases.

The sinus rate before right coronary arteriography and left coronary arteriography was similar (average values of 79.2 b.p.m. and 80.3 b.p.m.), with a coefficient of correlation of $0 \cdot 74$.

\section{DEGREE OF BRADYCARDIA}

The mean decrease of sinus rate after the injection of contrast medium into the right coronary artery was 16.6 b.p.m. and 16.9 b.p.m. after injection of the left coronary artery. Even with a similar decrease in sinus rate the coefficient of correlation was poor $(r=0 \cdot 4)$.

The degree of resultant bradycardia was greater in normal cases and in those with hypertrophic cardiomyopathy than in patients with valvular heart disease and congestive cardiomyopathy (Table 1). Patients with ischaemic heart disease showed an intermediate decrease of sinus rate. The smallest decrease was found during left coronary injection in patients with congestive cardiomyopathy and valvular heart disease.

The decrease of frequency caused by injection of the dominant artery was significantly greater than the decrease achieved by injection of the other artery (Fig. 2). We found no significant correlation with any of the other angiographic and haemodynamic values, such as the ejection fraction, the

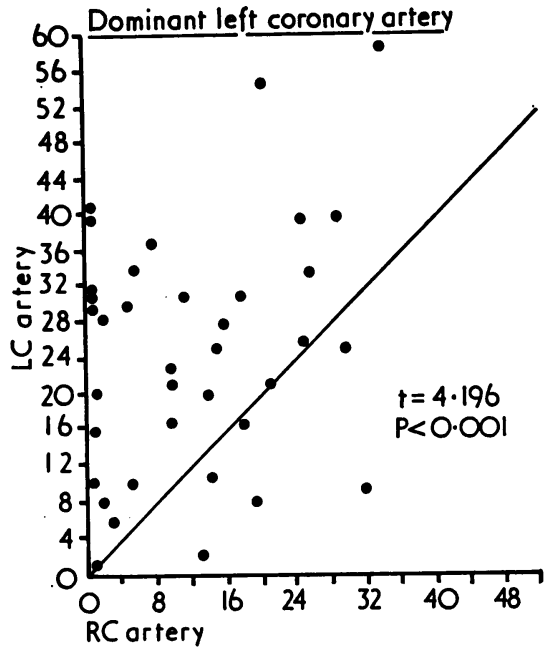

Fig. 3 Decrease of sinus rate during right coronary arteriography ( $R C$ artery) and left coronary arteriography ( $L C$ artery) in 37 cases with main perfusion to the inferior wall of the left ventricle provided by the left coronary artery.

end-diastolic left ventricular volume and pressure and the presence of left ventricular dyskinesia.

In cases with dominant left coronary artery the decrease of sinus rate caused by injection of this artery was 24.9 b.p.m. while that caused by injection of the right coronary artery was $11 \cdot 7$,

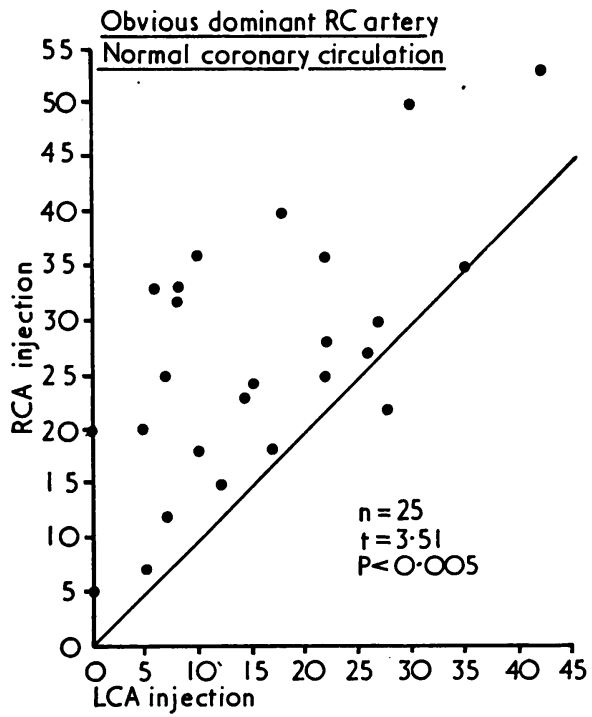

Fig. 4 Decrease of sinus rate during injection of the right coronary artery ( $R C A$ injection) and of the left coronary (LCA injection) in 25 cases with obvious dominant right coronary artery. 
Table 2 Influence of sinus node artery origin on decrease of sinus rate (beats per minute) during coronary arteriography

(a) Decrease of sinus rate during injection of dominant artery Origin of sinus node artery

$\begin{array}{ll}\text { Homolateral } & \begin{array}{l}\text { decrease } \\ 20.9\end{array} \\ \text { Contralateral } & 19.3\end{array} \quad \mathrm{t}=0.7, \mathrm{P}=0.45$

(b) Results in 63 cases with sinus node artery arising from left coronary artery Dominant artery

Left coronary Mean decrease

Right coronary

$\mathrm{t}=1 \cdot 48, \mathrm{P}=0.055$

(c) 101 cases of ischaemic heart disease in which the sinus node artery originated from the right coronary in $65 \%$ of them Injection into Mean

Left coronary artery

decrease

Right coronary artery

$18 \cdot 7$

$t=-1 \cdot 7, P=0.055$

(d) 32 cases with dominant left coronary artery and with sinus node artery originating from right coronary in 65 per cent

Injection into

Mean

Left coronary artery

Right coronary artery

24.9

$t=4 \cdot 2, P=0.001$

resulting in a highly significant statistical difference of $t=4.19(P<0.001)$ (Fig. 3).

In the group with dominant right coronary artery the mean decrease of rate caused by injection of this artery was 18.3 b.p.m. and that achieved by injection into the left was 15.3 b.p.m., a difference which fell just short of significance at the $5 \%$ level $(P=0.055)$. In the 25 cases with an obviously dominant right coronary artery (Fig. 4) the decrease of sinus rate was closely related to the injection of the dominant artery $(t=3.51)(P<0.001)$.

In the group of cases with balanced circulation to the inferior wall of the left ventricle, in which the atrioventricular node artery originated from the right coronary artery, the mean decrease of frequency caused by injection of the right coronary artery was similar to that caused by injection of the left coronary artery $(t=0.34$ and $P=0.75)$ (Fig. 5).

The mean decrease caused by injection of the coronary artery which gave origin to the sinus node artery was 18.7 b.p.m., compared with a decrease of 15.5 b.p.m. resulting from injection of the other artery $(t=2.1$ and $P=0.025)$. The same artery gave origin to both the artery which supplied the inferior wall of the left ventricle and the sinus node artery in 60 per cent of the cases. In order to evaluate further the influence of the sinus node artery we carried out several correlations (Table 2): (a) The mean decrease of sinus rate caused by injection of the dominant artery was 20.9 b.p.m. when this artery gave origin to the sinus node artery, and 19.8 b.p.m. when it did not (Table 2a), with no significant difference. (b) In the group of cases with the sinus node artery arising from the left coronary artery the mean decrease of sinus rate caused by injection of this artery was 26.5 b.p.m. when this artery perfused the inferior wall of the left ventricle and 18.2 b.p.m. when it did not $(P=0.055)$ (Table 2b). (c) In 101 cases of ischaemic heart disease in which we were able to determine the origin of the sinus node artery the mean decrease of sinus rate caused by injection of the left coronary artery was 18.7 b.p.m. while that caused by injection of the right coronary artery was 15.7 even when the sinus node artery originated from the right in 65 per cent of the cases (Table 2c). (d) In the group of cases with left dominance the degree of bradycardia was greater during left coronary arteriography even when the sinus node artery came from the right coronary artery in 65 per cent of the cases (Table 2d).

\section{SINUS ARREST}

Sinus arrest lasting longer than 2 seconds was found on 8 occasions while injecting into the left coronary artery and in 4 while injecting into the right coronary artery. One patient suffered arrest after injection of both arteries. The sinus arrest had no correlation with the origin of the sinus node artery, as it was produced in three cases while injecting into the coronary which gave origin to this artery and in 8 cases during injection of the other artery. In one case the origin of the sinus node artery could

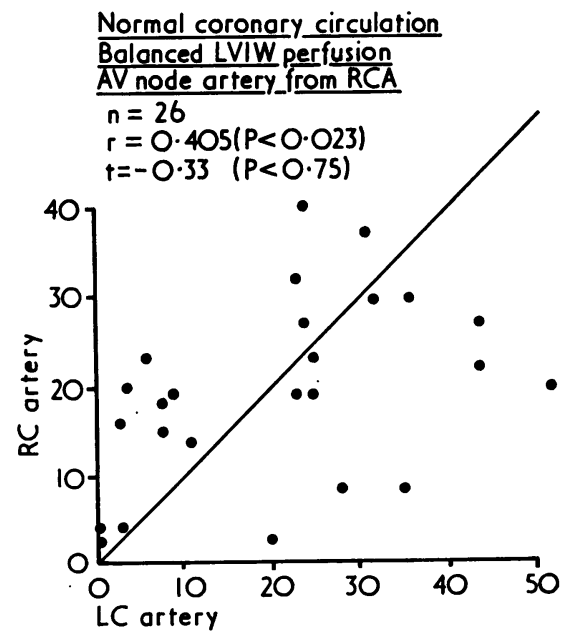

Fig. 5 Decrease of sinus rate during right coronary arteriography ( $R C$ artery) and left coronary arteriography (LC artery) in 26 cases with equilibrated circulation to the inferior wall of the left ventricle (LVIW perfusion). RCA, right coronary artery. 
Table 3 Episodes of sinus arrest longer than two seconds

\begin{tabular}{|c|c|c|c|c|c|c|}
\hline Arrest during injection of & $\begin{array}{l}\text { No. of } \\
\text { cases }\end{array}$ & $\begin{array}{l}\text { Origin of sinus } n \\
\text { Homolateral }\end{array}$ & $\begin{array}{l}\text { node artery } \\
\text { Contralateral }\end{array}$ & $\begin{array}{l}\text { Perfusion to infer } \\
\text { Homolateral }\end{array}$ & $\begin{array}{l}\text { rior wall of left } \\
\text { Contralateral }\end{array}$ & $\begin{array}{l}\text { ventricle } \\
\text { Balanced }\end{array}$ \\
\hline $\begin{array}{l}\text { Left coronary artery } \\
\text { Right coronary artery }\end{array}$ & $\begin{array}{l}8 \\
4\end{array}$ & $\begin{array}{l}2 \\
1\end{array}$ & $\begin{array}{l}6 \\
2\end{array}$ & $\begin{array}{l}5 \\
3\end{array}$ & 2 & $\begin{array}{l}1 \\
1\end{array}$ \\
\hline
\end{tabular}

not be identified. Arrest was more frequent during injection of the dominant artery ( 8 cases). The circulation was balanced in 2 (Table 3).

\section{Discussion}

Left ventricular reflexes have been studied by various techniques in animal experiments: ligature of one or more coronary arteries (Hanley et al., 1971, 1972; Thorén, 1972), injection of microspheres of mercury into the aortic root or selectively into a coronary artery (Kezdi et al., 1974), and inhalation of low concentrations of oxygen (Thorén, 1973a). Various factors, such as the type of anaesthesia used, thoracotomy, and the different surgical procedures, are probably the cause of lack of uniformity in their results. Furthermore, these studies are not repeatable in human beings.

The common response to coronary arteriography is characterised by bradycardia and vasodilatation, measurements of which have been used to assess the intensity of the reflex response. We have used the degree of bradycardia to study the ventricular parasympathetic reflexes.

Low heart rate is common in acute inferior myocardial infarction, as has been reported by Fluck et al. (1967), and Julian et al. (1964). The pathogenesis of this bradycardia has been speculated on: George and Greenwood (1967) conclude that it is not caused by interruption of the sinus node artery, and others, such as Adgey et al. (1968), give a predominant role to ischaemia of the coronary sinus region caused by obstruction to flow to the atrioventricular node artery and arteries of the crux. Our findings (Table 2 and Fig. 4) indicate that injection into the sinus node artery and the atrioventricular node artery does not influence the degree of bradycardia caused by coronary arteriography.

Patients with the bradycardia-hypotension syndrome secondary to acute inferior myocardial infarction also present signs of peripheral vasodilatation (Thomas and Woodgate, 1966; Kuhn, 1967), which, in the presence of prolonged hypotension, indicates failure of the baroreceptor reflex (Costantin, 1963; Kezdi et al., 1974; Thorén, 1973b).

VENTRICULAR REFLEXES

The inhibition of the baroreceptor reflex mechanism seems to result from the existence of a new reflex which originates from receptors situated in the left ventricle, as pointed out by Costantin (1963) and by Öberg and Thorén (1972). These receptors are sensitive to various stimuli: hypoxia, compression, distension, etc. Öberg and Thorén (1972) found greater quantities of receptors sensitive to mechanical stimulation in the anterolateral wall of the left ventricle and could not show pressure receptors in the wall of the right ventricle.

The majority of authors localise the ischaemic receptors in the wall of the left ventricle without making reference to their distribution. Some authors studied the reflexes from the posteroinferior wall by tying off or selectively injecting the circumflex artery with mercury (Kezdi et al., 1974; Costantin, 1963), but made no correlations with those produced by ischaemia of other areas of the left ventricle.

According to our results, the bradycardia produced by coronary arteriography results from reflexes which originate mainly in the inferior wall of the left ventricle. That was the almost invariable finding in cases with dominant left coronary artery (Fig. 3) and in those with an obviously dominant right coronary artery (Fig. 4). In some other cases the injection of the dominant right coronary artery resulted in a less pronounced bradycardia than that caused by the injection of the contralateral vessel. To explain this we suggest three reasons: (a) The presence of receptors in other zones of the ventricles, which can be deduced from the constant appearance of bradycardia after accidental selective injection of the anterior descending artery or of the conus branch of the right coronary artery. (b) The quantity of contrast medium and the velocity of the injection can influence the degree of bradycardia in individual cases. (c) By following the classic concept we included in this group cases with a large area of the inferior wall of the left ventricle perfused from the left coronary artery. It is not uncommon to see that branches of this artery (circumflex, posterolateral, and distal parts of the anterior descending artery) can compensate and sometimes overbalance the supply provided by the so-called dominant right coronary artery. Support for this is given by the comparison of our Fig. 4 and 5 which show a distinct difference in the degree of bradycardia 
caused by left coronary arteriography. The main angiographic difference between both groups lies in the importance and distribution of the circumflex and obtuse marginal arteries, which provide significant perfusion to the inferior wall of the left ventricle in the group with a balanced circulation; however, the circumflex artery was poorly developed and the obtuse marginal artery followed a lateral pathway in the other group.

Sinus arrest lasting more than 2 seconds has a clear relation with stimulus of the inferior wall of the left ventricle (Table 3) and it also stresses the role of this wall in the pathogenesis of sinus bradycardia during coronary arteriography.

Previous studies (Carson and Lazzara, 1970) have suggested the possibility that the reflexes caused by coronary arteriography are the result of dilatation of the coronary capillaries and small veins; this hypothesis was based on the ability to cause a similar response by blockage and distention of the coronary sinus. Further studies may be necessary to elucidate the cause of these reflexes with the view to evaluating the role of myocardial hypoxia, a factor not ruled out in the above-mentioned study.

\section{CONDUCTION PATHWAYS OF}

\section{VENTRICULAR REFLEXES}

The reflexes originating in the left ventricle are conducted centrally via the vagus nerve, a fact shown experimentally by abolition of their reflexes by cooling or sectioning of the nerves. None the less, Hanley et al. (1971) showed that though sectioning of the vagus nerve significantly decreases the reflex vasodilatation after coronary occlusion, it does not completely abolish it.

According to Kezdi et al. (1974), the carotid glomus may participate, since the activity of the efferent sympathetic nerve fibres is diminished during the hypotension which follows acute inferior cardiac infarction. This may justify the decrease of the habitual baroreceptor reflex and supports the role of the sympathetic nervous system in the efferent pathway of these reflexes. On the other hand, the participation of the parasympathetic system is not understood, because atropine does not block the vasodilatation produced by cardiac stimulus in experimental animal work (Costantin, 1963; Hanley et al., 1971, 1972; Kezdi et al., 1974), and it does not influence the peripheral vasodilatation produced by coronary arteriography according to Carson and Lazzara (1970); however, the last authors showed that atropine can completely eliminate the bradycardia produced by coronary arteriography.

We acknowledge the work of Dr. P. Zarco, who performed most of the coronary arteriograms, R. Carrera, a medical student in our service, for his assistance, and Drs. W. Brigden and $L$. McDonald for their help and criticism.

\section{References}

Adgey, A. A. J., Geddes, J. S., Mulholland, H. C., Keegan, D. A. J., and Pantridge, J. F. (1968). Incidence, significance, and management of early bradyarrhythmia complicating acute myocardial infarction. Lancet, 2, 1097-1101.

Agress, C. M., Glassner, H. F., Binder, M. J., and Fields, J. (1957). Hemodynamic measurements in experimental coronary shock. Fournal of Applied Physiology, 10, 469-475.

Carson, R. P., and Lazzara, R. (1970). Hemodynamic responses initiated by coronary stretch receptors with special reference to coronary arteriography. American fournal of Cardiology, 25, 571-578.

Costantin, L. (1963). Extracardial factors contributing to hypotension during coronary occlusion. American fournal of Cardiology, 11, 205-217.

Fluck, D. C., Oslen, E., Pentcost, B. L., Thomas, M., Fillmore, S. J., Shillingford, J. P., and Mounsey, J. P. D. (1967). Natural history and clinical significance of arrhythmias after acute cardiac infarction. British Heart fournal, 29, 170-189.

George, M., and Greenwood, T. (1967). Relation between bradycardia and the site of the myocardial infarction. Lancet, 2, 739-740.

Hanley, H. G., Costin, J. C., and Skinner, N. S. (1971). Differential reflex adjustments in cutaneous and muscle vascular beds during experimental coronary artery occlusion. American fournal of Cardiology, 27, 513-521.

Hanley, H. G., Raizner, A. E., Inglesby, T. V., and Skinner, N. S. (1972). Response of the renal vascular bed to acute experimental coronary arterial occlusion. American fournal of Cardiology, 29, 803-808.

Judkins, M. P. (1968). Percutaneous transfemoral selective coronary arteriography. Radiologic Clinics of North America, 6, 467-492.

Julian, D. G., Valentine, P. A., and Miller, G. C. (1964). Disturbances of rate, rhythm and conduction in acute myocardial infarction. American fournal of Medicine, 37, 915-927.

Kezdi, P., Kordenat, R. K., and Misra, S. N. (1974). Reflex inhibitory effects of vagal afferents in experimental myocardial infarction. American fournal of Cardiology, 33, 853-860.

Kuhn, L. A. (1967). Treatment of cardiogenic shock. American Heart fournal, 74, 578-581.

Linden, R. J. (1975). Reflexes from the heart. Progress in Cardiovascular Diseases, 18, 201-221.

Öberg, B., and Thorén, P. (1972). Studies on left ventricular receptors, signalling non-medullated vagal afferents. Acta Physiologica Scandinavica, 85, 145-163.

Sleight, P., and Widdicombe, J. G. (1965). Action potentials in fibres from receptors in the epicardium and myocardium of the dog's left ventricle. Fournal of Physiology, 181, 235-258.

Sones, F. M., and Shirey, E. K. (1962). Cine coronary arteriography. Modern Concepts of Cardiovascular Disease, 31, 735-738.

Thomas, M., and Woodgate, D. (1966). Effect of atropine on bradycardia and hypotension in acute myocardial infarction. British Heart fournal, 28, 409-413.

Thorén, P. (1972). Left ventricular receptors activated by 
severe asphyxia and by coronary artery occlusion. Acta Physiologica Scandinavica, 85, 455-463.

Thorén, P. (1973a). Reflex bradycardia elicited from left ventricular receptors during acute severe hypoxia in cats. Acta Physiologica Scandinavica, 87, 103-112.

Thorén, P. (1973b). Evidence for a depressor reflex elicited from left ventricular receptors during occlusion of one coronary artery in the cat. Acta Physiologica Scandinavica, 88, 23-34.

Requests for reprints to Dr. F. Perez-Gomez, Departamento Cardiopulmonar, Hospital Clinico de la Facultad de Medicina, Madrid, Spain. 